\title{
Avaliação de capacitação para utilização do Protocolo NICHD em duas cidades brasileiras
}

\author{
NICHD Protocol training assessment in two Brazilian cities
}

\author{
Chayene Hackbarth, Lúcia Cavalcanti de Albuquerque Williams e Nahara Rodrigues Laterza Lopes
}

Universidade Federal de São Carlos, São Paulo, Brasil

\begin{abstract}
Resumen: Considerando recomendações de boas práticas forenses na escuta de crianças, pesquisadores do National Institute of Child Health and Human Development (NICHD), nos E.U.A, desenvolveram o Protocolo de Entrevista estruturada NICHD. O presente trabalho tem como objetivo avaliar uma capacitação oferecida a 15 profissionais (psicólogas e assistentes sociais) de dois municípios brasileiros. A capacitação envolveu um curso teórico presencial sobre o protocolo em dois dias e uma parte prática que compreendia realizar, gravar e enviar em áudio para as pesquisadoras no mínimo uma entrevista realizada antes e outra entrevista após a capacitação. Tais entrevistas foram analisadas e comparadas, sendo fornecido feedback à distância sobre o desempenho aos participantes. Adicionalmente, as participantes completaram o Questionário de Aceitação Social sobre a capacitação. Após a capacitação com o protocolo, houve um aumento significativo no uso de perguntas abertas nas entrevistas realizadas, bem como aumento significativo da taxa de detalhes de relevância forense nas respostas às questões diretas. A avaliação dos participantes foi, em geral, positiva. Limitações do estudo referem-se ao número reduzido de participantes e de entrevistas realizadas, bem como problemas intrínsecos ao contexto forense brasileiro, com interferência de perguntas diretas e sugestivas por operadores de Direito durante o Depoimento Especial da criança.
\end{abstract}

Palabras clave: Protocolo NICHD, entrevistas forenses, abuso sexual infantil, oitiva de crianças.
Abstract: Following recommendations of good forensic practice in children's hearing, researchers from the National Institute of Child Health and Human Development (NICHD), USA, developed the NICHD structured interview Protocol. This study aims to evaluate a training program offered to 15 professionals (psychologists and social workers), in two Brazilian municipalities. The training involved a two-day workshop on theoretical issues associated with the protocol, and a practical component which required participants to send researchers at least one audio recorded interview conducted without and another interview after the training with the NICHD Protocol. These interviews were analyzed and compared, and feedback about participant's performance was provided to them through online sessions. Participants also completed the Social Acceptance Questionnaire giving opinions on the training received. After the protocol training, a significant increase in the use of openended questions in the interviews with NICHD Protocol was observed, as well as in the rate of details of forensic relevance in children's answers to directive questions. Participant training evaluation was generally positive. Study limitations involve the small number of participants and interviews, as well as problems intrinsic to the Brazilian forensic system, with interference of directive and suggestive questions by attorneys during crossexamination in the child's testimony.

Keywords: NICHD protocol, forensic interview, child sexual abuse, child hearing.

Agradecimento à Fundação de Amparo e Apoio à Pesquisa de São Paulo (Processo No 2013/06690-9).

Contacto: Centro de Educação e Ciências Humanas, Programa de Pós-Graduação em Psicologia, Laboratório de Análise e Prevenção da Violência. Rod. Washington Luís, km 235, São Carlos, São Paulo, Brasil 13565-905. Correo electrónico: laprev_ufscar@yahoo.com.br

Cómo citar: Hackbarth, C., Williams, L. C. A. y Lopes, N. R. L. (2015). Avaliação de capacitação para utilização do Protocolo NICHD em duas cidades brasileiras. Revista de Psicología, 24(1), 1-18.

http://dx.doi.org/10.5354/0719-0581.2015.36916 


\section{Introdução}

O abuso sexual infantil (ASI) é um fenômeno relativamente frequente que não tem recebido a devida atenção do sistema judiciário brasileiro para a punição do agressor e proteção das crianças envolvidas. Um dos desafios encontrados para a solução dos casos são as dificuldades inerentes no sentido de mostrar evidências de que o fenômeno tenha ocorrido, o que, somadas às lacunas da legislação e despreparo de profissionais que atuam com casos de ASI, resultam no fato de o ofensor não receber uma punição adequada (Cezar, 2007; Williams, 2009).

Outras dificuldades apontadas para a solução dos casos de ASI consistem na repetição de entrevistas com crianças; a lentidão do processo; o estresse vivenciado pelas vítimas e suas famílias; a exposição e o constrangimento a que são submetidas; a proximidade com o ofensor na audiência; bem como o desamparo que sentem ao depor em espaço isolado dos demais familiares de confiança (Santos \& Gonçalves, 2008; Williams, 2009). Dentre os desafios apontados para a comprovação do abuso estão as exigências de exames médicos confirmatórios, os quais apresentam controvérsias jurídicas, sendo considerados de valor limitado para a comprovação da agressão (Goodman, 1984; Herman, 2009). Assim, há décadas, pesquisadores têm investigado o assunto, atestando que as supostas vítimas são muitas vezes a única fonte de informação disponível e empreendendo esforços consideráveis para entender como o testemunho das crianças pode ser feito da forma mais precisa possível (Malloy \& Quas, 2009).

Tais esforços são meritórios, considerando as consequências negativas à vida dos envolvidos em casos de suspeita de ASI, quando os profissionais falham em identificar a sua ocorrência. Nestes casos o tratamento necessário à criança ou a assistência à família não são oferecidos e, o que é ainda mais preocupante, o abuso pode continuar ocorrendo (Faust, Bridges \& Ahern, 2009). Além disso, nos casos em que o abuso sexual é confirmado em sentença, mas não ocorreu, há sofrimento desnecessário e irreparável por parte da criança, de sua família e do suspeito ofensor (O'Donohue \& Fanetti, 1996).

Katz, Barnetz e Hershkowitz (2014) afirmaram ser importante desenvolver ferramentas profissionais para garantir que a investigação do abuso não seja uma experiência traumática, bem como causa do aumento de ansiedade, estresse e sentimento de culpa da criança. Os autores afirmam que, ao contrário do que é, a investigação deveria ser uma experiência de empoderamento da criança, proporcionando sentimentos de confiança, autoestima e justiça. De acordo com Wood e Garven (2000), entrevistas mal conduzidas podem criar oportunidades para que os advogados de defesa acusem o profissional de ter realizado uma entrevista sugestiva e enganosa. Os autores complementam que os erros mais graves cometidos pelos entrevistadores podem ser eliminados com o devido treinamento sobre a realização adequada de entrevistas investigativas.

Mediante a importância da oitiva da criança para a condução de um processo que não a revitimize e, considerando que não há uma maneira confiável de confirmar se houve ou não ASI, baseando-se exclusivamente na existência de sintomas que sejam prevalentes apenas em crianças vítimas de ASI, ou seja, sintomas que nunca seriam diagnosticados em crianças que não foram vítimas de ASI (Faust et al., 2009), profissionais diver- 
sos vêm realizando oitivas infantis com a utilização de protocolos investigativos.

O Protocolo NICHD foi desenvolvido no National Institute of Child Health and Human Development, instituto americano que lhe deu o nome, por Lamb, Hershkowitz, Orbach e Esplin (2008). Tal protocolo foi construído com base nos pressupostos da Entrevista Cognitiva (Fischer \& Geiselman, 1992), atendendo recomendações de boas práticas indicadas para as entrevistas forenses com questões padronizadas a serem seguidas pelo entrevistador, além do treino que auxilia a criança na diferenciação entre verdade e mentira (Lamb et al., 2008). O Protocolo NICHD se sobressai, dentre outros protocolos de entrevista investigativa para crianças, pelo número de estudos empíricos realizados que evidenciaram os benefícios em sua utilização para a oitiva em casos de suspeita de ASI (Aldridge et al., 2004; Hershkowitz, Horowitz, Lamb, Orbach \& Sternberg, 2004; Hershkowitz, Horowitz, \& Lamb, 2005; Hershkowitz, Orbach, Lamb, Sternberg \& Horowitz, 2006; Hershkowitz, Lanes \& Lamb, 2007; Hershkowitz, Fisher, Lamb \& Horowitz, 2007; Lamb et al., 2008; Lamb \& Garretson, 2003; Lamb, Sternberg, Orbach, Hershkowitz \& Horowitz, 2003; Lamb, Orbach, Hershkowitz, Esplin \& Horowitz, 2007; Malloy, Brubacher \& Lamb, 2011; Orbach et al., 2000; Peixoto, Ribeiro e Alberto, 2013; Snider \& Everson, 2011).

De acordo com Lamb et al. (2008) e Toth (2011), as recomendações para a utilização do protocolo são extensas, porém os profissionais forenses frequentemente apresentam dificuldades em aderir às práticas recomendadas nesse campo de atuação. Por esse motivo, os autores do Protocolo NICHD desenvolveram um roteiro de entrevista estruturado no qual as recomendações profissionais são estabelecidas por orientações operacionais definidas. Não há outro protocolo que se compare em termos de validação empírica para os fins a que se propõe e de credibilidade para analisar a veracidade dos relatos (Herman, 2009). O Protocolo NICHD é amplamente utilizado em países como os Estados Unidos da América, Suécia, Finlândia, Noruega, Canadá, Reino Unido e Israel, tendo já sido avaliado em outros países como Portugal (ver Williams, Hackbarth, Blefari, Padilha \& Peixoto, 2014 para uma revisão de estudos realizados em tais países).

Compreendendo a necessidade de capacitar profissionais brasileiros para a realização de oitivas de crianças em casos de suspeita de ASI e considerando a existência de uma versão traduzida do Protocolo NICHD para o contexto brasileiro (Williams et al., 2012), capacitações com este protocolo e estudos para sua validação vêm sendo recentemente realizados no Brasil. Em uma dissertação de mestrado (Blefari, 2014), o uso do protocolo foi avaliado por meio de delineamento de sujeito único com três profissionais no Estado do Paraná, tendo mostrado resultados promissores. Adicionalmente, em estudo piloto realizado pelas presentes autoras foram encontrados resultados encorajadores após uma capacitação oferecida a sete profissionais do Sudeste do Brasil que jamais haviam realizado oitiva de crianças com protocolos estruturados (Hackbarth, Williams \& Lopes, em preparo). Entretanto, em tal estudo piloto não foi possível comparações entre entrevistas realizadas antes e depois da capacitação com o Protocolo NICHD, logo a necessidade de se investigar a hipótese da efetividade de se capacitar profissionais brasileiros para a realização de entrevistas investigativas 
com o protocolo NICHD, de acordo com as recomendações de boas práticas nas entrevistas forenses.

\section{Objetivo}

Avaliar a efetividade de uma capacitação a psicólogos e assistentes sociais, no sentido de aumentar a qualidade das entrevistas forenses realizadas. O objetivo último do estudo consiste em fornecer evidências da utilidade e viabilidade da utilização do Protocolo NICHD para o contexto brasileiro.

\section{Método}

\section{Triagem dos participantes}

Após parceria firmada com o Ministério Público da cidade A, a promotora de Justiça recomendou a capacitação sobre o Protocolo NICHD a todos os profissionais da Rede de Proteção à Criança e ao Adolescente do município. Adicionalmente, seis profissionais do Fórum Judiciário e uma psicóloga clínica de outro Estado do país demonstraram interesse e foram a tal cidade para realizar a capacitação. Cinquenta profissionais compareceram à parte teórica da capacitação, sendo que 33 profissionais fizeram-na como ouvintes e 17 assinaram o Termo de Consentimento Livre e Esclarecido (TCLE) para participação no estudo. Os critérios para inclusão no presente estudo eram: participação em todos os encontros da capacitação; entrega de gravação, em formato de vídeo ou áudio, de, no mínimo, uma entrevista realizada em casos de suspeita de ASI sem o uso do Protocolo NICHD (antes da capacitação) e outra realizada com o uso deste protocolo (após a capacitação). Considerando a indisponibilidade de casos com suspeita de ASI na Rede de Proteção da cidade A, foram também aceitas entrevistas reali- zadas em casos de suspeita de abuso físico uma vez que o protocolo também se presta para tal finalidade (Hershkowitz et al., 2005; Hershkowitz et al., 2006).

Considerando a baixa adesão ao estudo na cidade $A$, foi realizada uma segunda capacitação por meio de parceria estabelecida com o Fórum de uma cidade localizada em Estado e região distinta (cidade B). Todos os 13 psicólogos e assistentes sociais do Fórum Judicial foram convidados e aceitaram participar. Adicionalmente, dois Juízes de Direito assistiram a capacitação na condição de ouvintes.

\section{Participantes}

Participaram do estudo 15 profissionais do sexo feminino, sendo duas participantes da cidade A (uma psicóloga clínica e psicóloga do Fórum Judiciário) e 13 participantes da cidade B (nove psicólogas e quatro assistentes sociais do Fórum Judiciário).

\section{Local}

$\mathrm{Na}$ cidade $\mathrm{A}$, a capacitação foi realizada na Fundação Escola do Ministério Público. $\mathrm{Na}$ cidade $\mathrm{B}$, a capacitação foi realizada em uma sala de formação continuada no Fórum Judicial.

\section{Instrumentos}

Protocolo de Entrevista Investigativa NICHD. Ao ser desenvolvido, o Protocolo NICHD teve como objetivo traduzir as recomendações e o estado da arte de pesquisas de forma a facilitar a evocação por crianças de relatos de supostos casos de abuso que fossem completos e acurados (Orbach et al., 2000). Tal meta é alcançada, segundo tais autores, criando-se um ambiente de entrevis- 
ta acolhedor (fase pré-substantiva de estabelecimento de rapport); adaptandose a entrevista para o nível de desenvolvimento da criança (minimizando a complexidade linguística e evitando interrupções); preparando a criança para a tarefa a ser realizada de fornecer informações (esclarecendo regras de comunicação, treinando a criança a relatar eventos específicos que envolvam a memória episódica), e maximizando perguntas do entrevistador que evoquem o relato livre. Assim, o Protocolo NICHD (Lamb et al., 2008) é constituído por perguntas abertas, utilizadas de acordo com os relatos apresentados pela criança na maior parte da sessão. $\mathrm{O}$ uso de perguntas sugestivas é evitado, diminuindo a possibilidade de contaminação de seus relatos. O protocolo é composto por duas partes distintas, a parte présubstantiva e a parte substantiva. A parte pré-substantiva é subdividida nas seguintes etapas: etapa introdutória; construção do vínculo; e treino para a memória episódica. A parte substantiva apresenta, em sua maior parte, questões abertas, especialmente para os relatos da criança sobre o abuso, como a pergunta principal: "Me conta tudo sobre isso". A fim de esclarecer alguns pontos relatados pela criança na entrevista, o protocolo apresenta perguntas diretas e de múltipla escolha, a serem utilizadas de acordo com a necessidade do entrevistador e o relato da criança. A entrevista é finalizada com um tópico neutro. $\mathrm{O}$ protocolo utilizado no presente estudo consistiu na versão final ${ }^{1}$ elaborada por Lamb e colaboradores (2008) e traduzida para o português (versão brasileira) por Williams, Hackbarth, Blefari e Padilha (2012).

\footnotetext{
${ }^{1} \mathrm{O}$ instrumento pode ser acessado em sua íntegra em http://nichdprotocol.com/nichdbrazil.pdf
}

Questionário de Aceitação Social dos Profissionais. Tal questionário foi elaborado pelas autoras com o intuito de verificar se há evidências da utilidade e viabilidade da utilização do Protocolo NICHD para o contexto brasileiro de acordo com a avaliação realizada pelos participantes. Este instrumento é constituído por duas partes: a primeira referente à avaliação dos participantes sobre o conteúdo teórico e à avaliação geral dos participantes sobre a capacitação, e a segunda referente à avaliação dos participantes sobre a parte prática com o uso do Protocolo NICHD. A primeira parte é composta por uma questão em que o participante é solicitado a atribuir uma nota de zero a 10 para a capacitação recebida (justificando sua nota) e por 10 afirmações em que deve avaliar o seu grau de concordância com as mesmas de acordo com uma escala Likert de 4 pontos. Neste sentido, eram apresentadas aos participantes afirmações como "Os conceitos trabalhados foram relevantes" e esses deveriam responder de acordo com uma das seguintes opções: Concordo totalmente, Concordo, Discordo e Discordo totalmente. Nesta parte, há, ainda, uma questão em que os participantes devem avaliar se recomendariam ou não a capacitação para outro colega de profissão e outra em que podem oferecer sugestões para próximas capacitações. A segunda parte é composta por cinco questões, sendo uma de múltipla escolha e quatro questões abertas. Na questão de múltipla escolha o participante deve avaliar o sentimento gerado durante a condução da entrevista utilizando o protocolo NICHD de acordo com as seguintes opções de resposta: "seguro, inseguro, constrangido, confortável, objetivo, perdido, orientado e outros". Finalmente, as questões abertas foram: "Considera que as revelações das crianças apresentaram dados confiáveis a respeito da situação abusi- 
va?"; "Foi possível encontrar maior número de detalhes na fala das crianças em comparação às entrevistas realizadas antes da capacitação?"; e "Comentários sobre o feedback realizado após as entrevistas com o Protocolo NICHD”.

\section{Procedimento}

Considerações éticas. O estudo foi aprovado pelo Comitê de Ética em Pesquisa em Seres Humanos (processo $\mathrm{N}^{\mathrm{o}}$ 15583813.2.0000.5504), tendo todos os participantes assinado o Termo de Consentimento Livre e Esclarecido.

Capacitação do Protocolo NICHD. As capacitações realizadas tiveram como modelo o treinamento de cinco dias intensivos com base no estudo desenvolvido em Quebec, no Canadá, por Cyr e
Lamb (2009), bem como um estudo piloto realizado pelas autoras com psicólogos de um Estado da região Sudeste do Brasil (Hackbarth et al., em preparo). A capacitação teórica, realizada presencialmente, compreendeu dois dias intensivos de trabalho com oito horas de duração cada. As apresentações orais do conteúdo foram realizadas pelas autoras com recursos multimídia. As principais referências utilizadas para planejar a capacitação teórica envolveram os estudos realizados por Brown e Lamb (2009); Cunningham (2009); Harris, Goodman, Augusti, Chae e Alley (2009); Klemfuss e Ceci (2009); Lamb et al. (2007; 2008); La Rooy, Lamb e Pipe (2009); Malloy e Quas (2009); Williams (2014) e Williams et al. (2014). O conteúdo das sessões teóricas da capacitação encontra-se sumariado na tabela 1 .

\section{Tabela 1}

Conteúdo programático da parte teórica da capacitação

\begin{tabular}{|c|c|c|}
\hline Período & Tema & Atividade \\
\hline \multicolumn{3}{|l|}{ Dia 1} \\
\hline \multirow[t]{3}{*}{ Manhã } & Os desafios do trabalho em Rede & Dinâmicas e aula dialogada \\
\hline & Introdução sobre protocolos de entrevista & $\begin{array}{l}\text { sobre revisão teórica da litera- } \\
\text { tura }\end{array}$ \\
\hline & $\begin{array}{l}\text { Efeitos da sugestionabilidade nas entrevistas } \\
\text { forenses }\end{array}$ & $\begin{array}{l}\text { Exibição de trechos do filme: } \\
\text { A Caça (Vinterberg, 2012) }\end{array}$ \\
\hline Tarde & $\begin{array}{l}\text { Peculiaridades do desenvolvimento infantil } \\
\text { (memória, processos cognitivos e capaci- } \\
\text { dades comunicativas em crianças) } \\
\text { Memórias traumáticas e falsas memórias }\end{array}$ & $\begin{array}{l}\text { Apresentação do conteúdo oral } \\
\text { dialogado e discussão }\end{array}$ \\
\hline \multicolumn{3}{|l|}{ Dia 2} \\
\hline Manhã & $\begin{array}{l}\text { O Protocolo NICHD: objetivos e fases } \\
\text { Entrevistas realizadas no Brasil com o Pro- } \\
\text { tocolo NICHD }\end{array}$ & $\begin{array}{l}\text { Aula dialogada e esclarecimen- } \\
\text { to de dúvidas } \\
\text { Apresentação de atendimentos } \\
\text { em áudio }\end{array}$ \\
\hline Tarde & Aplicação prática do Protocolo NICHD & $\begin{array}{l}\text { Simulação do uso (Role- } \\
\text { playing em duplas alternando } \\
\text { papel entrevistador/criança) }\end{array}$ \\
\hline
\end{tabular}


Concluída a capacitação teórica, as participantes foram instruídas a conduzir ao menos uma entrevista com o Protocolo NICHD, encaminhando sua gravação em áudio ou vídeo às pesquisadoras. Os procedimentos de oitiva com crianças eram realizados de modo similar em ambos os Fóruns Judiciários (cidades A e B), de acordo com a Recomendação No 33 de 23 de Novembro de 2010, que discorre sobre o modelo de Depoimento Especial para a escuta de crianças e adolescentes vítimas ou testemunha de violência nos processos judiciais (Conselho Nacional de Justiça, 2010). Como os profissionais da cidade $\mathrm{B}$ alegaram não ser possível fazer um intervalo durante a oitiva em sua prática forense (procedimento padrão do Protocolo NICHD), foi solicitado aos juízes ouvintes do curso colaboração no sentido de autorizar tal intervalo. De acordo com a orientação do protocolo, este intervalo tem como objetivo propiciar o efeito de reminiscência na memória da criança sobre possíveis fatos abusivos, bem como o planejamento das próximas perguntas a serem realizadas pelo entrevistador.

Finalmente foi realizada uma sessão individual para fornecer feedback sobre as entrevistas realizadas após a capacitação. As sessões de feedback foram realizadas por meio de videoconferências, conforme disponibilidade e preferência das participantes. Cada sessão de feedback teve duração de uma hora, ocasião em que foram discutidas as particularidades das entrevistas realizadas pela participante (aspectos positivos e negativos quanto ao uso do protocolo), além do esclarecimento de dúvidas. Ao término da sessão era aplicado o Questionário de Avaliação Social.

\section{Análise de dados}

Para análise dos dados obtidos com o Protocolo NICHD foi utilizado o seu Manual de Codificação desenvolvido por Orbach e Lamb (não publicado) e utilizado amplamente nas pesquisas com este protocolo (ver por exemplo, Aldridge et al., 2004; Lamb et al., 1996; Cederborg, Orbach, Sternberg \& Lamb, 2000; Orbach et al., 2000; Cyr \& Lamb, 2009; Malloy et al., 2011; Katz \& Hershkowitz, 2012; Lamb, Hershkowitz \& Lyon, 2013). O Manual categoriza as perguntas como sendo abertas ("Me conta tudo sobre isso"); diretas ("Quantos anos ele tem?"); de múltipla escolha ("Ele passou a mão por cima ou por baixo da sua roupa?"); e sugestivas ("Ele tomou banho com você, não tomou?"). Desta forma, como proposto no Manual de Codificação disponibilizado para pesquisadores (Orbarch \& Lamb, não publicado) foram contabilizadas a frequência de perguntas utilizadas nas entrevistas de acordo com sua categoria (abertas, sugestivas, diretas ou de múltipla escolha); a frequência de palavras utilizadas pelas supostas vítimas em seus relatos; e a frequência de detalhes relevantes gerados a partir das questões apresentadas nas entrevistas realizadas antes e após a capacitação.

Os detalhes de relevância forense foram computados a partir da análise minuciosa das informações obtidas nos relatos das crianças. Um detalhe é considerado relevante quando oferece qualquer informação relacionada ao abuso e que tenha sido comunicada pelo entrevistado durante a entrevista. Neste sentido, o detalhe é respaldado pela identificação ou caracterização de uma pessoa, objeto, 
evento, local, ação, emoção, pensamento sensação relacionados ao suposto abuso (Orbach \& Lamb, não publicado).

Com o objetivo de verificar qual tipo de questão ocasionou o maior número de detalhes de relevância forense, foi calculada a taxa de detalhes dividindo-se o número de detalhes obtidos por cada tipo de pergunta pelo número de perguntas realizadas. Esta taxa fornece uma comparação de efetividade com relação à obtenção de detalhes. Para determinar se a utilização do Protocolo NICHD promoveu a obtenção de um maior número de relatos de abuso, maior extensão na fala da criança a respeito do evento e maior número de detalhes de relevância forense e considerando o tamanho reduzido da amostra, foram realizados os testes estatísticos não paramétricos de Wilcoxon e de Friedman.

Para análise do Questionário de Aceitação Social foi calculada a média aritmética da nota atribuída pelos profissionais à capacitação. As demais informações do questionário foram analisadas qualitativamente, observando-se o conteúdo das respostas fornecidas nas questões abertas.

\section{Resultados}

Os participantes realizaram 20 entrevistas antes da capacitação, sendo que nove participantes realizaram uma entrevista, três participantes realizaram duas entrevistas e um participante realizou três. Uma entrevista se referiu a um caso em que houve revelação de abuso físico e 19 estavam relacionadas a casos com suspeita de ASI, sendo que em duas delas não houve revelação de abuso. Para as entrevistas realizadas antes da capacitação, a idade média das crianças entrevistadas foi de $9.92(D P=0.941)$ anos, tendo a idade variado de 5 a 15 anos.
Após a capacitação, foram realizadas 16 entrevistas, sendo que 15 participantes realizaram apenas uma entrevista e uma participante realizou duas. Todas as entrevistas após a capacitação foram relacionadas a casos de suspeita de ASI e apenas uma não obteve revelação de abuso. A idade média das crianças entrevistadas após a capacitação foi de $10.67(D P=0.70)$, variando de 7 a 16 anos. Vale destacar que não foi observada diferença significativa na idade das crianças entrevistadas antes da capacitação sem o Protocolo NICHD $(M e=9)$ e após a capacitação com o mesmo $(M e=$ 11) $\left(S^{+}=5.13 ; S^{-}=5.75 ; Z=-0.715 ; p\right.$ $=0.474 ; N=12)$.

Para as análises estatísticas, foram consideradas apenas as entrevistas com revelação de abuso físico ou sexual entregues pelos participantes antes e após a capacitação. Neste sentido, três participantes foram excluídos da análise, pois uma de suas entrevistas (duas realizadas sem o uso do protocolo e uma com) não contemplava a revelação de abuso, totalizando 12 participantes cujas entrevistas foram analisadas. Nos casos em que o participante entregou mais de uma entrevista com revelação referente ao mesmo momento de avaliação (antes ou após a capacitação) foi utilizada a média do número de detalhes e palavras obtidas e de perguntas realizadas em cada entrevista.

A tabela 2 apresenta a mediana e a frequência para o uso de perguntas abertas, diretas, de múltipla escolha e sugestivas, bem como os resultados do teste de Wilcoxon, utilizado para comparar a diferença das medidas entre os dois momentos de avaliação, ou seja, com e sem o uso do Protocolo NICHD. 
Tabela 2

Mediana de frequência de perguntas abertas, diretas, de múltipla escolha e sugestionáveis realizadas com e sem o Protocolo NICHD

\begin{tabular}{lcccccc}
\hline & \multicolumn{2}{c}{\begin{tabular}{c} 
Sem protocolo \\
\multicolumn{1}{c}{$(n=12)$}
\end{tabular}} & \multicolumn{2}{c}{ Com protocolo } \\
\multicolumn{1}{c}{ Perguntas } & $M e$ & $\%$ & $M e$ & $\%$ & $Z$ & \multicolumn{1}{c}{ M } \\
\hline Abertas* & 2.0 & 3.7 & 6.5 & 13.6 & -2.226 & .026 \\
Diretas & 28.75 & 44.7 & 24.25 & 46.6 & -0.471 & .638 \\
Múltipla Escolha & 20.25 & 41.8 & 13.50 & 31.4 & -1.571 & .116 \\
Sugestivas & 5.50 & 9.8 & 3.50 & 8.4 & -1.023 & .306 \\
\hline
\end{tabular}

Nota: $\mathrm{O} n=$ refere-se ao número de participantes em cada momento de avaliação. $* p<.05$

Como pode ser observado na tabela 2, apesar de a porcentagem de utilização de perguntas abertas ainda ser modesta nas entrevistas com o Protocolo NICHD (13.6\%), observou-se um aumento significativo se comparada à utilização de tais perguntas sem o protocolo (3.7\%). Em relação às demais modalidades de perguntas não houve alterações com ou sem o uso do protocolo. Quando considerados o número total de detalhes e de palavras obtidas nas entrevistas, independente do tipo de pergunta utilizada, não foi observada diferença significativa para a frequência de detalhes antes da capacitação $(M e=30.5)$ e após $(M e=24.5)(Z=-0.981 ; p=.327) \mathrm{e}$ para a frequência de palavras sem $(M e=$ 833.50) e com o uso do protocolo $(\mathrm{Me}=$ 781.50) $(Z=-0.628 ; p=.530)$.

Foi observado, ainda, que a psicóloga clínica (cidade A) e uma das profissionais do Fórum Judiciário (cidade B) não utilizaram qualquer tipo de pergunta sugestiva após a capacitação, sendo que a primeira também não utilizou perguntas sugestivas antes da capacitação.

Para avaliar se houve diferença entre o número de detalhes de acordo com o tipo de pergunta utilizada (taxa de detalhes) antes e após a capacitação foi utilizado o teste estatístico de Friedman. A tabela 3 apresenta a mediana para a taxa de detalhes obtida sem e com o uso do Protocolo NICHD para cada tipo de pergunta.

Tabela 3

Mediana da taxa de detalhes obtida sem e com o uso do protocolo de acordo com o tipo de pergunta

\begin{tabular}{lcc}
$\begin{array}{l}\text { Taxa de } \\
\text { detalhes }\end{array}$ & $\begin{array}{c}\text { Sem } \\
\text { protocolo } \\
(n=12)\end{array}$ & $\begin{array}{c}\text { Com } \\
\text { protocolo } \\
(n=12)\end{array}$ \\
\hline Abertas & .00 & .44 \\
Diretas & .53 & $.57^{*}$ \\
Múltipla & .31 & .39 \\
escolha & & \\
Sugestivas & .11 & .00 \\
\hline
\end{tabular}

Nota: $\mathrm{O} n=$ refere-se ao número de participantes em cada momento de avaliação. $* p<.05$

Como pode ser observado na tabela 3 , houve um aumento significativo na taxa de detalhes obtidos com as perguntas diretas após a capacitação, visto que a diferença crítica entre os dois momentos de avaliação foi maior do que $37.48(\mathrm{Me}=$ 0.53 antes; $M e=0.57$ após) quando utilizado o teste de Friedman $\left(X^{2}(7)=\right.$ 20.063; $p=.005 ; n=12$ ). 
Cabe destacar que 28 entrevistas foram realizadas de acordo com o procedimento de Depoimento Especial, tendo sido observada durante as transcrições a interferência dos operadores de direito durante 26 destas entrevistas. Diferentemente do acordado, essas perguntas não ocorreram apenas para o profissional durante o intervalo (sem a presença da criança) e sim ao longo da entrevista. Estas interferências ocorreram por meio de diversas perguntas diretas, de múltipla escolha e sugestivas reproduzidas pelos participantes às crianças durante o Depoimento Especial.

Questionário de Aceitação Social dos Profissionais. A nota média oferecida pelas participantes à capacitação foi 8.0 $(S D=0.75)$, tendo variado de 7.0 a 9.5. Todas as participantes responderam que recomendariam a capacitação para um colega de profissão e todas afirmaram que, após a capacitação, a revelação das crianças apresentou dados confiáveis sobre a situação abusiva.

Em relação ao sentimento gerado durante a condução das entrevistas com o Protocolo NICHD, nove participantes apontaram ter se sentido orientadas. Nas justificativas apresentadas, as participantes comentaram que a capacitação foi interessante e proveitosa, parabenizando o desempenho, a didática e o domínio do conteúdo por parte das facilitadoras.

Dentre os pontos negativos apontados pelas participantes, cinco relataram o fato de o protocolo "parecer mecânico" e 11 apontaram o fato de o protocolo "exigir que o profissional realize uma entrevista mais curta". Em relação a este último ponto, a dificuldade das participantes em realizar uma entrevista mais curta estaria relacionada ao fato de os operadores de direito incluírem rotineiramente perguntas diretas, de múltipla escolha e sugestivas nas salas de audiência, fazendo-as reproduzir tais perguntas à criança na sala do Depoimento Especial.

Ainda sobre esta questão, duas participantes comentaram que "o protocolo não abarca os limites relacionados ao livre contraditório", caracterizado pelas perguntas diretas, de múltipla escolha e sugestivas realizadas pelo Defensor Público na sala de audiências. Três participantes também relataram "preocupação sobre a impossibilidade de se fazer o intervalo para elaboração das perguntas abertas", o que prejudicaria a reminiscência na memória da criança sobre possíveis fatos abusivos e o planejamento das próximas perguntas, conforme orientação do protocolo.

Em relação às perguntas sobre o conteúdo teórico ministrado, 12 participantes (todas da cidade B) concordaram parcialmente quando questionadas sobre os encontros terem oferecido oportunidade para obter conhecimento sobre a sugestionabilidade no depoimento de crianças vítimas de ASI, bem como sobre a memória, processos cognitivos e capacidades comunicativas da criança. A queixa apresentada por todas foi a respeito do "curto tempo de capacitação para aprofundar a discussão sobre a temática apresentada", sendo que as participantes mencionaram, ainda, ter "conhecimento prévio dos assuntos discutidos na capacitação".

Vale ressaltar que as participantes da cidade $\mathrm{B}$ não consideraram que o número de detalhes de relevância forense foi maior nos relatos das crianças com a utilização do Protocolo NICHD, contrariando os resultados do presente estudo em relação a maior frequência de detalhes nas perguntas diretas realizadas com o protocolo. Estas afirmam ter utilizado outros instrumentos (Entrevista Cognitiva, EC; e o roteiro NCAC, National Children Advocacy Cen- 
ter) para a realização das oitivas, os quais, segundo as mesmas, possibilitaram maior número de detalhes no relato da criança, relatos esses que se mostraram mais apropriados, em alguns casos, por atender as exigências do contexto forense brasileiro. Adicionalmente, as participantes da cidade B alegaram que o roteiro do Protocolo NICHD é similar ao do Protocolo NCAC).

Assim, 11 participantes da cidade B afirmaram que "o Protocolo NICHD não abarca todas as questões necessárias para esclarecer os fatos do suspeito abuso" e 10 participantes (as duas da cidade A e oito da cidade B), complementaram, ainda, que este "precisa ser reestruturado para que o layout seja mais apropriado, facilitando a busca da próxima pergunta a ser feita", de acordo com a necessidade do entrevistador e relato da criança.

Três participantes da cidade B sugeriram, ainda, que o protocolo fosse:

adaptado, apresentando uma pergunta direta específica para solicitar que a criança nomeie a parte intima do corpo à qual se refere quando relata sobre o abuso, uma vez que se a criança não a nomeia, há risco de anulação do processo judicial.

Em relação à fase de encerramento do protocolo que recomenda deixar um telefone com a criança se essa necessitar entrar em contato futuramente, duas participantes argumentaram que "não é possível deixar o contato profissional para a criança, visto que não permanecem em comunicação com esta posteriormente à entrevista".

Todas as participantes relataram como a principal dúvida "seguir as questões do protocolo na íntegra ou ter a possibilidade de flexibilizá-lo com perguntas pertinen- tes no momento da entrevista" e 10 participantes (uma da cidade A e nove da cidade B) afirmaram "ter a impressão de estar sendo repetitivo/insistente nas questões realizadas durante o treino da memória episódica". Contudo, todas as participantes concordaram sobre a possibilidade de tornar as perguntas flexíveis, sem sugestionar os relatos das crianças, após maior tempo de treinamento e prática com o instrumento.

\section{Discussão}

Esse estudo teve como objetivo avaliar se uma capacitação do Protocolo NICHD a profissionais brasileiros aumentaria a qualidade de entrevistas forenses realizadas. Em relação ao objetivo proposto, após a capacitação, observou-se um aumento significativo de perguntas abertas realizadas com o Protocolo NICHD, em comparação às perguntas realizadas sem o protocolo. Adicionalmente, foi constatado que o percentual de detalhes de relevância forense nas perguntas diretas foi significativamente maior quando utilizadas o mesmo tipo de questões após a capacitação com o referido protocolo.

Os resultados acima reforçam estudos extensos defendendo que o uso do Protocolo NICHD em oitiva de crianças pode alterar favoravelmente as condições para a recuperação da memória, independente de fatores como a idade e resistência para fazer o relato de ASI e/ou abuso físico. Mais especificamente, as entrevistas com o protocolo apresentaram mais perguntas abertas do que as entrevistas realizadas com outros instrumentos, garantindo que as informações obtidas por meio das memórias episódicas recordadas fossem mais acuradas quando comparadas às memórias de reconhecimento, obtidas pelas perguntas focadas ou sugestivas, tal como sugerido por Fanetti, O'Donohue e 
Bradley, 2006; Hershkowitz et al., 2007; Lamb et al., 1996, 2007, 2008; Sternberg, Lamb, Davies e Westcott, 2001; Sternberg, Lamb, Orbach, Esplin e Mitchell, 2001. Tal comprovação é primordial quando as crianças são entrevistadas em âmbito jurídico, uma vez que as informações frequentemente são disponibilizadas somente pelo relato da criança ou do suspeito ofensor (Goodman, 1984; Lamb et al., 2008; Withcomb, 2003).

$\mathrm{O}$ aumento significativo para a taxa de detalhes obtidos por meio de perguntas diretas após a capacitação não confirma a hipótese de Lamb et al. (2008), de que as crianças forneceriam mais detalhes de relevância forense quando utilizadas questões abertas. Isto possivelmente ocorreu porque as perguntas abertas, no presente estudo, ainda que significativamente superiores com o Protocolo NICHD em comparação a sem o uso do protocolo, foram utilizadas em menor taxa do que o esperado, quando comparadas às demais categorias de perguntas, corroborando resultados de Hackbarth, et al. (submetido) no estudo piloto realizado com o protocolo.

Vale ressaltar, ainda, que devido à experiência prévia das participantes em realizar oitivas com outros protocolos ou roteiros (com a Entrevista Cognitiva e o roteiro de entrevista NCAC), não houve diferença significativa entre os momentos antes e após a capacitação na análise da extensão dos relatos das crianças, denotando que as entrevistas realizadas anteriormente compreendiam relatos relativamente extensos por parte da criança. Contudo, observouse que tais relatos foram contaminados por perguntas focadas e sugestivas, influenciados pelos operadores de Direito, como previamente informado. Além do mais, foi constatado que as participantes apresentaram resistência em aderir ao roteiro do Protocolo NICHD para a reali- zação das entrevistas devido à prática anterior com os demais instrumentos.

O presente estudo apresenta diversas limitações, sendo a primeira referente ao número reduzido de participantes e do número de entrevistas realizadas por estes (antes e após a capacitação), inviabilizando a possibilidade de análises mais robustas. Além disso, o fato de 26 dentre 28 entrevistas realizadas antes e após a capacitação ter sofrido interferência dos operadores de direito, que exigiam a utilização de perguntas focadas e sugestivas ao final da entrevista, não só sugere a contaminação dos relatos da criança, como impossibilita uma noção mais acurada do conhecimento prático dos participantes sobre o uso do protocolo. Por essa razão, não foi possível seguir completamente as normas de boas práticas para a realização das entrevistas forenses, que permitiriam que as participantes obtivessem um número maior de detalhes de relevância forense quando utilizadas as perguntas abertas, tipo de pergunta mais aconselhada, por evitar a indução dos relatos da criança durante a entrevista, especialmente quando se trata de crianças pequenas. Esta hipótese pode ser confirmada pelo uso excessivo das perguntas diretas, perguntas de múltipla escolha e perguntas sugestivas no estudo.

De acordo com Benia, Hauck-Filho, Dillenburg e Stein (2015), Davies, Westcott e Horan, (2000), Lamb et al. (2008) e Phillips, Oxburgh, Gavine e Myklebust (2012), o protocolo é mais efetivo no auxílio aos entrevistadores no sentido de colocar em prática as recomendações para a realização de entrevistas forenses, obtendo informações mais acuradas e evitando perguntas que suscitam informações pouco confiáveis. Contudo, tais benefícios só podem ser observados se o protocolo for aplicado integralmente. 
Corroborando Pelisoli, Gava e Dell'Aglio (2011); Pelisoli, Dobke e Dell'Aglio (2014); Powell, Wright e Clark (2010) tal prática dos operadores de direito nas oitivas realizadas no Brasil pode refletir um desconhecimento sobre os avanços científicos na área forense, o qual exigiria sensibilização e treinamento extensivo para conhecimento dos riscos associados às perguntas focadas e sugestivas para a contaminação dos relatos da criança. Por fim, os desafios relacionados à contaminação dos relatos da criança sobre o suposto abuso, observados pelos profissionais que realizaram a oitiva no modelo do Depoimento Especial, carecem de uma reflexão ampla do Sistema Judiciário brasileiro de modo que seja possível enfrentá-los. Entretanto, é importante fazer a ressalva de que um dos juízes ouvintes da capacitação realizada na cidade $\mathrm{B}$ cumpriu o que foi acordado durante as aulas, realizando o intervalo previsto pelo protocolo durante o Depoimento Especial e atestando a possibilidade de modificar o andamento da audiência, de acordo com as recomendações de boas práticas nas entrevistas forenses (Lamb et al., 2008).

Outra limitação do presente estudo a ser destacada, tampouco intrínseca ao protocolo e sim à Rede de Proteção brasileira, foi que, corroborando Santos e Gonçalves (2008) e Williams e colaboradores (2014), os profissionais das duas cidades constataram o fenômeno de repetição excessiva de entrevistas com as supostas vítimas por diferentes serviços e profissionais dos municípios. Tal prática foi observada uma vez que cinco crianças que já haviam relatado o abuso para outros profissionais apresentaram resistência durante a oitiva com o Protocolo NICHD e dificuldades para recordar o evento, devido ao tempo decorrido entre a denúncia e a escuta especial. Tal sequela de repetição de entrevistas foi semelhante ao que também ocorreu no estudo piloto anteriormente mencionado (Hackbarth, et al., submetido).

A limitação final consiste no fato de que, de acordo com relatos de 13 participantes da cidade $\mathrm{B}$, tais profissionais já utilizavam outros instrumentos ou roteiros para a realização de oitivas. Neste sentido, o desempenho de tais profissionais no protocolo ficou contaminado por tal experiência prévia e não se sabe qual seria o desempenho das mesmas sem tal experiência preliminar. Tais profissionais pontuaram que o roteiro de entrevista NCAC será implantado na cidade $\mathrm{B}$, de acordo com determinação do Conselho Nacional de Justiça, evidenciando a necessidade de se realizar estudos para avaliar comparativamente a qualidade das entrevistas realizadas com o Protocolo NICHD e com o Protocolo NCAC, notadamente, pois não há pesquisas publicadas sobre o último.

A respeito da não utilização de perguntas sugestivas, tanto pela psicóloga clínica da cidade A, quanto pela participante do Fórum Judiciário da cidade B, observouse a possibilidade de se realizar uma entrevista livre de perguntas que contaminem o relato da criança, quando essa é realizada individualmente e sem a interferência de outros profissionais, ou, ainda, quando há a possibilidade de reformular as perguntas realizadas pelos operadores de direito na sala de audiências, permitindo o livre relato ou simplesmente referindo-se exclusivamente a informações previamente trazidas pela criança em seus relatos. Verificou-se que a participante da cidade $\mathrm{A}$, que não apresentou questões sugestivas antes ou após a capacitação, seguiu o roteiro do Protocolo NICHD mais rigorosamente. Tal profissional não tinha experiência prévia em realizar oitivas de crianças, fato que pode ter sido positivo por evitar que utilizasse outras 
técnicas concomitantemente. Além disso, é possível que, dada a autonomia para realizar a entrevista individualmente com a criança em um contexto no qual não havia interferências externas, a participante tenha tido mais facilidade para seguir as orientações oferecidas durante a capacitação. Resultados com diminuição expressiva na contaminação dos relatos da criança têm sido apresentados em um trabalho recente de Cederborg, Alm, Nises e Lamb (2012).

Novos estudos necessitam ser realizados em outras regiões brasileiras, a fim de identificar as peculiaridades do sistema judiciário concernentes às localidades nas quais não são realizadas oitivas de crianças ou nos casos em que estas são realizadas sem a utilização de um instrumento estruturado para tal. Adicionalmente, a utilização de outros instrumentos (EC e NCAC) necessitam de análises comparativas com o uso do Protocolo NICHD para avaliar qual destes é mais apropriado para a investigação de casos de suspeita de ASI.

A fim de implantar no sistema judiciário brasileiro um modelo de oitiva em sala de Depoimento Especial, conforme indicado pelas boas práticas para a realização das entrevistas forenses, é relevante que os profissionais da área do Direito recebam orientações e cursos que os sensibilizem e informem a respeito das características inerentes ao processo de investigação de ASI, qualificando-os para realizar o trabalho em parceria com a equipe multidisciplinar e evitando prejuízos para a solução dos casos julgados, bem como para a saúde emocional da suposta vítima (Pelisoli \& Dell'Aglio, 2014). Por fim, se faz evidente a necessidade de realizar novos estudos brasileiros sobre o Protocolo NICHD, ampliando o seu conteúdo prático e incluindo sessões mais frequentes de feedback e supervisão mais demorada, de forma a diminuir o uso de questões diretas, questões de múltipla escolha e, sobretudo, perguntas sugestivas, elevando expressivamente o uso de perguntas abertas.

O presente trabalho enfatiza a pertinência e o caráter inovador do estudo no contexto desenvolvido apontando que há ainda um longo caminho a trilhar para que a criança brasileira não sofra contínuas revitimizações ao ser repetidamente entrevistada e, para que o Sistema Judiciário Brasileiro se adeque às boas práticas de oitivas, apesar de esforços recentes. Ainda que modestos, os resultados do presente estudo atestam que é possível colaborar para tal caminhada.

\section{Referências}

Aldridge, J., Lamb, M. E., Sternberg, K. J., Orbach, Y., Esplin, P. W., \& Bowler, L. (2004). Using a human figure drawing to elicit information from alleged victims of child sexual abuse. Journal of Consulting and Clinical Psychology in the Public Domain, 72(2), 304-316.

Benia, L. R., Hauck-Filho, N., Dillenburg, M., \& Stein, L. M. (2015). The NICHD investigative interview protocol: A meta-analytic review. Journal of Child Sexual Abuse, 24(3), 259-279.

http://dx.doi.org/10.1080/10538712.2015.1006749 
Blefari, C. A. (2014). Capacitação de profissionais para o uso do Protocolo NICHD em entrevistas investigativas de abuso sexual infantil (Dissertação de mestrado). Universidade Tuiutí do Paraná, Curitiba.

Brown, D. A. \& Lamb, M. E. (2009). Forensic interviews with children. A two-way street: Supporting interviewers in adhering to best practice recommendations and enhancing children's capabilities in forensic interviews. In K. Kuehnle \& M. Connell (Eds.), The evaluation of child sexual abuse allegations. A comprehensive guide to assessment and testimony (pp. 299-309). Hoboken, NJ: Wiley.

Cezar, J. A. D. (2007). Depoimento sem dano: uma alternativa para inquirir crianças e adolescentes nos processos judiciais. Porto Alegre: Livraria do Advogado.

Cederborg, A. C., Orbach, Y., Sternberg, K. J., \& Lamb, M. E. (2000). Investigative interviews of child witnesses in Sweden. Child Abuse \& Neglect, 24(10), 1355-1361.

Cederborg, A. C., Alm, C., Nises, D. L. S., \& Lamb, M. E. (2012). Investigative interviewing of alleged child abuse victims: An evaluation of a new training program for investigative interviewers. Police Practice and Research: An International Journal, 14(3), 242-254.

Conselho Nacional de Justiça. (2010). Recomendação $N^{o} 33$ de 23 de novembro de 2010. Brasília: CNJ.

Cyr, M. \& Lamb, M. E. (2009). Assessing the effectiveness of the NICHD investigative interview protocol when interviewing French-speaking alleged victims of child sexual abuse in Quebec. Child Abuse \& Neglect, 33(5), 257-268.

Cunningham, A. (2009). Dificuldades apresentadas por crianças ao prestar depoimento no Fórum Judicial e como ajudá-las. Em L. C. A. Williams \& E. A. C. Araújo (Orgs.), Prevenção do abuso sexual infantil: um enfoque interdisciplinar (pp. 162-167). Curitiba: Juruá.

Davies, G. M., Westcott, H. L., \& Horan, N. (2000). The impact of questioning style on the content of investigative interviews with suspected child sexual abuse victims. Psychology, Crime \& Law, 6(2), 81-97.

Fanetti, M., O’Donohue, W., \& Bradley, A. (2006). A method for evaluating child forensic interviews. American Journal of Forensic Psychology, 24(3), 5-27.

Faust, D., Bridges, A. J., \& Ahern, D. C. (2009). Methods for the identification of sexually abused children. In K. Kuehnle \& M. Connell (Eds.), The evaluation of child sexual abuse allegations. A comprehensive guide to assessment and testimony (pp. 3-19). Hoboken, NJ: Wiley.

Fisher, R. P. \& Geiselman, R. E. (1992). Memory-enhancing techniques for investigating interviewing: The cognitive interview. Springfield, MA: Charles C. Thomas.

Goodman, G. S. (1984). Children's testimony in historical perspective. Journal of Social Issues, 40(2), 9-31.

Hackbarth, C., Williams, L. C. A., \& Lopes, N. R. L. (2015). Investigação de abuso sexual infantil com o Protocolo NICHD: um estudo piloto. Manuscrito submetido para publicação. 
Harris, L. S., Goodman, G. S., Augusti, E. M, Chae, Y., \& Alley, D. (2009). Children's resistance to suggestion. In K. Kuehnle \& M. Connel (Orgs.), The evaluation of child sexual abuse allegations. A comprehensive guide to assessment and testimony (pp. 182198). Hoboken, NJ: Wiley.

Herman, S. (2009). Forensic child sexual abuse evaluations. Accuracy, ethics, and admissibility. In K. Kuehnle \& M. Connel (Eds.), The evaluation of child sexual abuse allegations. A comprehensive guide to assessment and testimony (pp. 247-255). Hoboken, NJ: Wiley.

Hershkowitz, I., Horowitz, D., Lamb, M. E., Orbach, Y., \& Sternberg, K. J. (2004). Interviewing youthful suspects in alleged sex crimes: A descriptive analysis. Child Abuse \& Neglect, 28(4), 423-438.

Hershkowitz, I., Horowitz, D., \& Lamb, M. E. (2005). Trends in children's disclosure of abuse in Israel: A national study. Child Abuse \& Neglect, 29(11), 1203-1214.

Hershkowitz, I., Orbach, Y., Lamb, M. E., Sternberg, K. J., \& Horowitz, D. (2006). Dynamics of forensic interviews with suspected abuse victims who do not disclose abuse. Child Abuse \& Neglect, 30(7), 753-769.

Hershkowitz, I., Lanes, O., \& Lamb, M. E. (2007). Exploring the disclosure of child sexual abuse with alleged victims and their parents. Child Abuse \& Neglect, 31(2), 111-123.

Hershkowitz, I., Fisher, S., Lamb, M. E., \& Horowitz, D. (2007). Improving credibility assessment in child sexual abuse allegations: The role of the NICHD investigative interview protocol. Child Abuse \& Neglect, 31(2), 99-110.

Katz, C. \& Hershkowitz, I. (2012). The effect of multiple art prompts on children's testimonies in sexual abuse investigations. Child Abuse \& Neglect, 36(11-12), 753-759.

Katz, C., Barnetz, Z., \& Hershkowitz, I. (2014). The effect of drawing on children's experiences of investigations following alleged child abuse. Child Abuse \& Neglect, 38(5), 858-867.

Klemfuss, J. Z. \& Ceci, S. (2009). Normative memory development and the child witness. In K. Kuehnle \& M. Connel (Orgs.), The evaluation of child sexual abuse allegations. A comprehensive guide to assessment and testimony (pp. 153-173). Hoboken, NJ: Wiley.

Lamb, M. E., Hershkowitz, I., Sternberg, K. J., Esplin, P. W., Hovav, M., Manor, T., \& Yudilevitch, L. (1996). Effects of investigative utterance types on Israeli children's responses. International Journal of Behavioral Development, 19(3), 627-637.

Lamb, M. E. \& Garretson, M. E. (2003). The effects of interviewer gender and child gender on the informativeness of alleged child sexual abuse victims in forensic interviews. Law and Human Behavior, 27(2), 71-157.

Lamb, M. E., Sternberg, K. J., Orbach, Y., Hershkowitz, I., \& Horowitz, D. (2003). Differences between accounts provided by witnesses and alleged victims of child sexual abuse. Child Abuse \& Neglect, 27(9), 1019-1031.

Lamb, M. E., Orbach, Y., Hershkowitz, I., Esplin, P. W., \& Horowitz, D. (2007). A structured forensic interview protocol improves the quality and informativeness of investigative interviews with children: A review of research using the NICHD Investigative Interview Protocol. Child Abuse \& Neglect, 31(11), 1201-1231. 
Lamb, M. E., Hershkowitz, I., Orbach, Y., \& Esplin, P. W. (2008). Tell me what happened: Structured investigative interviews of child victims and witnesses. Wiley Series in Psychology of Crime, Policing and Law. Hoboken, NJ: Wiley Blackwell.

Lamb, M. E., Hershkowitz, I., \& Lyon, T. D. (2013). Interviewing victims and suspected victims who are reluctant to talk. APSAC Advisor, 25(4), 16-19.

La Rooy, D., Lamb, M. E., \& Pipe, M. E. (2009). Repeated interviewing: A critical evaluation of the risks and potential benefits. In K. Kuehnle \& M. Connel (Orgs.), The evaluation of child sexual abuse allegations. A comprehensive guide to assessment and testimony (pp. 182-198). Hoboken, NJ: Wiley.

Malloy, L. C. \& Quas, J. A. (2009). Children's suggestibility: Areas of consensus and controversy. In K. Kuehnle \& M. Connel (Orgs.), The evaluation of child sexual abuse allegations. A comprehensive guide to assessment and testimony (pp. 267-287). Hoboken, NJ: Wiley.

Malloy, L. C., Brubacher, S. P., \& Lamb, M. E. (2011). Expected consequences of disclosure revealed in investigative interviews with suspected victims of child sexual abuse. Applied Developmental Science, 15(1), 8-19.

O’Donohue, S. \& Fanetti, M. (1996). Assessing the occurrence of child sexual abuse: An information processing, hypothesis testing approach. Aggression and Violent Behavior, $1(3), 269-281$.

Orbach, Y., Hershkowitz, I., Lamb, M. E., Sternberg, K. J., Esplin, P. W., \& Horowitz, D. (2000). Assessing the value of structured protocols for forensic interviews of alleged child abuse victims. Child Abuse \& Neglect, 24(6), 733-752.

Orbach, Y. \& Lamb, M. E. (2015). Quality of interview content analysis of investigative interviews codebook. Manuscrito inédito.

Peixoto, C. E., Ribeiro, C., \& Alberto, I. (2013). O Protocolo de Entrevista Forense do NICHD: contributo na obtenção do testemunho da criança no contexto português. Revista do Ministério Público, 134, 181-219.

Pelisoli, C., Gava, L. L., \& Dell'Aglio, D. D. (2011). Psicologia jurídica e tomada de decisão em situações envolvendo abuso sexual infantil. Psico-USF, 16(3), 327-338.

http://dx.doi.org/10.1590/S1413-82712011000300009

Pelisoli, C. \& Dell'Aglio, D. D. (2014). As contribuições da psicologia para o sistema de justiça em situações de abuso sexual. Ciência e Profissão, 34(4), 916-930.

Pelisoli, C., Dobke, V., \& Dell'Aglio, D. D. (2014). Depoimento especial: para além do embate e pela proteção das crianças e adolescentes vítimas de violência sexual. Temas em Psicologia, 22(1), 25-38.

http://dx.doi.org/10.9788/TP2014.1-03

Phillips, E., Oxburgh, G., Gavin, A., \& Myklebust, T. (2012). Investigative interviews with victims of child sexual abuse: The relationship between question type and investigation relevant information. Journal of Police and Criminal Psychology, 27(1), 45-54. 
Powell, M., Wright, R., \& Clark, S. (2010). Improving the competency of police officers in conducting investigative interviews with children. Police Practice and Research, 11(3), 211-226.

Santos, B. R. \& Gonçalves, I. B. (2008). Depoimento sem medo (?) culturas e práticas nãorevitimizantes: uma cartografia das experiências de tomada de depoimento especial de crianças e adolescentes. São Paulo, SP: Childhood Brasil.

Snider, S. M. \& Everson, M. D. (2011). What is my next question? Using question frameworks to improve children's narrative accounts of abuse. APSAC Advisor, 4(2), 11.

Sternberg, K. J., Lamb, M. E., Davies, G. M., \& Westcott, H. L. (2001). The memorandum of good practice: Theory versus application. Child Abuse \& Neglect, 25(5), 669-681.

Sternberg, K. J., Lamb, M. E., Orbach, Y., Esplin, P. W., \& Mitchell, S. (2001). Use of a structured investigative protocol enhances young children's responses to free-recall prompts in the course of forensic interviews. Journal of Applied Psychology, 86(5), 997-1005.

Toth, P. J. D. (2011). Comparing the NICHD and RATAC child forensic interview approaches - do the differences matter? Official Newsletter of the International Society for Prevention of Child Abuse and Neglect (ISPCAN), 20(1). Recuperado de http://c.ymcdn.com/sites/www.ispcan.org/resource/resmgr/link/ispcan_link_20.1.pdf

Vinterberg, T. (Produtor). (2012). A Caça [DVD]. Suécia: Califórnia Filmes.

Williams, L. C. A. (2009). Introdução ao estudo do abuso sexual infantil e análise do fenômeno no município de São Carlos. Em L. C. A. Williams \& E. A. C. Araújo (Eds.), Prevenção do abuso sexual infantil: um enfoque interdisciplinar (pp. 21-37). Curitiba, PR: Juruá.

Williams, L. C. A., Hackbarth, C., Blefari, C. A., \& Padilha, M. G. S. (2012). Guia de Entrevista Forense NICHD. Versão Português - Brasil. Recuperado de http://nichdprotocol.com/the-nichd-protocol/

Williams, L. C. A. (2014). Virginia Woolf's history of sexual victimization: A case study in light of current research. Psychology, 5, 1151-1164.

Williams, L. C. A, Hackbarth, C., Blefari, C. A., Padilha, M. G. S., \& Peixoto, C. E. (2014). Investigação de suspeita de abuso sexual infantojuvenil: o Protocolo NICHD. Temas em Psicologia, 22(2), 1-18.

Withcomb, D. (2003). Legal interventions for child victims. Journal of Traumatic Stress, $16(2), 149-157$.

Wood, J. M., \& Garven, S. (2000). How sexual abuse interviews go astray: Implications for prosecutors, police and child protection services. Child Maltreatment, 52(2), 109-129. 